\title{
Path Loss Prediction in Wireless Communication System using Fuzzy Logic
}

\author{
Sanu Mathew*, K. Shylaja, T. Jayasri and M. Hemalatha \\ School Of Computing, SASTRA University, Thanjavur, Tamil Nadu, India; \\ sanumathew03@gmail.com,shylu2518@gmail.com, jayasri5591@gmail.com; hemalatha@it.sastra.edu
}

\begin{abstract}
The portable wireless technology with wide accessibility facilitates the people to enter into a new dynamic environment for optimizing the overall productivity. Establishing reliable communication is a challenging aspect, because the signal propagation is heavily subjected to interference and fading effects resulting in severe path loss. In the field of telecommunication system, the effect of Path Loss in the signal is used to analyze and design the link budget system. Previously, many path loss prediction models like HATA, and Okumura are proposed where the path loss is determined with the help of experimental field values and verified with the help of the model graph. In this work, BPSK modulated signal is used to determine the path loss using HATA empirical formula derived with the help of the Okumura curve for various urban, suburban and rural areas. The identified values are given to triangular membership function and defuzzification is performed using faster and flexible center of sum method. The inferred results show maximum loss in case of urban and increases at an average rate of $10 \mathrm{~dB}$ per decade with distance. The proposed technique optimizes signal transmission by determining the path loss accurately.
\end{abstract}

Keywords: Binary Phase Shift Keying, Fuzzy Logic, HATA Model, Okumura Model, Path Loss

\section{Introduction}

The world is facilitated by advanced wireless technology for exchanging information between wireless gadgets in a relatively easy way. The design of wireless systems in telecommunication industries aims to enhance reliability of wireless interface for lossless transmission. One of the main characteristics that affect the transfer of information between the devices is the Propagation effect on the signal. The propagating signal quality is mainly degraded by interference and fading effects. The Propagation effect also includes Shadowing, Reflection, Refraction, Diffraction and Antenna Height. The influence on signal varies with respect to the environment in which they are present. Hence, Path Loss due to signal propagation in wireless medium has become a serious problem in the present world. Therefore, for an effective wireless communication, it is essential to understand the Path Loss of a signal between the transmitter and the receiver under different environmental conditions ${ }^{1}$.

Various Models are Present that deals with understanding the indoor and outdoor propagation of signal and the effect of Path Loss on it. Most of these models outline a basic method that enables us to study the effect of Path Loss in signal with respect to different terrain. These models make use of the field data to accurately predict the Path Loss effect on the signal. However, when these models are used in areas other than for which they are defined the obtained Path Loss data of signal is unreliable and untrustworthy. The Okumura curves are known for their practical use in the prediction of the Path Loss effect on the signal. HATA's empirical formula provided an efficient way for the prediction of Path Loss in different terrain. In most of the cases Path Loss models were used to monitor the Path Loss effect on signals in terrains such as Urban, Sub Urban and Rural.

${ }^{*}$ Author for correspondence 
In this proposal a modulated signal is generated and noise is added to incorporate the effect of the Path Loss to that signal. This signal is used to measure how it behaves in a different environment with the help of HATA Formulation and it is shown graphically with the help of Okumura Curve. The graph obtained from the generated values is compared with that of the model graph which shows how Path Loss occurs in the case of a modulated signal.

\section{HATA Okumura Path Loss Prediction Model}

The HATA model developed from Okumura includes graphical statistics of Okumura in order to identify the propagation effect of radio signals in the field of telecommunication for rural, urban and suburban trains. Okumura is one of the most widely employed Path Loss prediction model in frequencies ranging from $150 \mathrm{MHZ}$ to $1920 \mathrm{MHZ}^{2,3}$. Okumura came up as a set of curves shows how the signal behaves in different terrain regions and the effect of path loss on that signal in those terrains. But the Okumura curve is unable to adapt itself to the rapid changes that occur in terrain. This model is fairly good for the Urban and Sub Urban areas and it shows the poor efficiency while considering rural areas.

HATA make use of the Path Loss data from the Okumura curve and develops an empirical formula for predicting the effect of Path Loss in terrains such as Urban, Sub Urban and Rural. The HATA model can be used in the frequency ranging from $150 \mathrm{MHZ}$ to $1500 \mathrm{MHZ}$. Thus HATA empirical formula is derived from urban, subur- ban and rural areas with the help of Okumura statistics ${ }^{2,4}$. The standard formula for urban areas is given by

$$
\begin{array}{r}
L_{50}(\text { Urban })(d b)=69.55+26.16 \log f_{c}-13.82 \log h_{t e} \\
-a\left(h_{r e}\right)+\left(44.9-6.55 \log h_{t e}\right) \log d
\end{array}
$$

The standard formula for Sub Urban area is given by

$L_{50}(\operatorname{Sub} \operatorname{Urban})(d b)=L_{50}($ Urban $)-2\left[\frac{\log f_{c}}{28}\right]-5.4$

The standard formula for Rural area is given by

$$
\begin{aligned}
L_{50}(\text { Rural })(d b) & =L_{50}(\text { Urban })-4.48\left(\log f_{c}\right)^{2} \\
& -18.33 \log f_{c}-40.98
\end{aligned}
$$

Where $f_{c}$ is the frequency which has to be adjusted according to the area, $\mathrm{d}$ is the distance between the transmitter and the receiver, $h_{t e}$ is the height of the transmitter, $\mathrm{h}_{\mathrm{re}}$ is the height of the receiver ${ }^{5}$.

The effect of Path Loss in signals subjected to above mentioned terrains are predicted with the correction factor which has to be taken into account when they are used for Path Loss prediction. Most of the regions around the world make use of the empirical formula of HATA model for effective placements of the transmitter and the receiver.

The HATA model graph in Figure 1 shows the estimated path loss in large urban, suburban and rural areas. The influence of path loss steadily increases with respect to the distance between base stations. From the Figure 1, we can also infer that the environment in which the signal propagates is also a major factor because the path loss curve obtained for large urban is comparatively larger than the curves obtained for suburban and rural areas. Apart

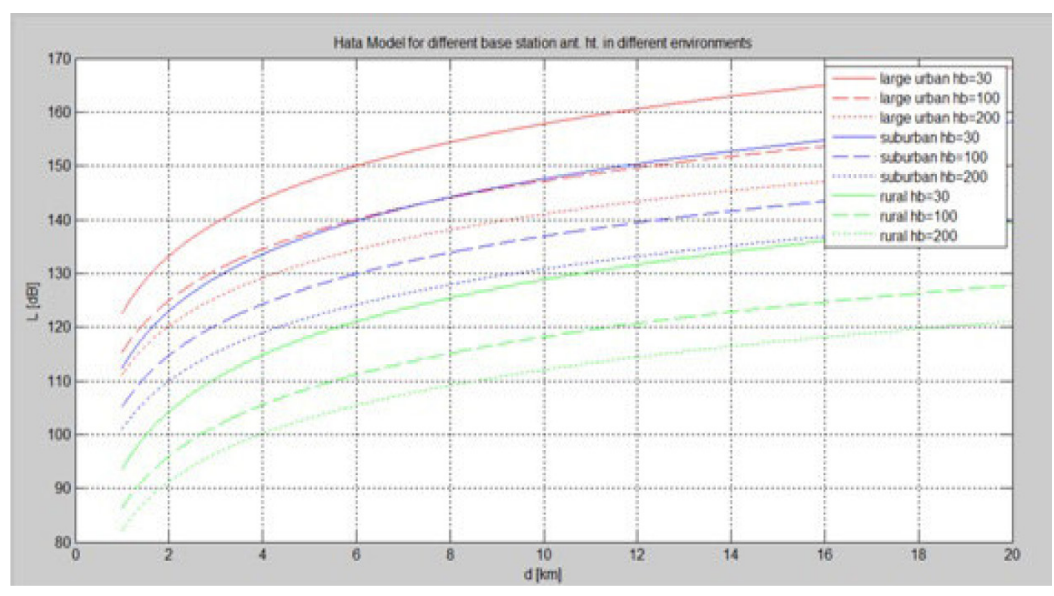

Figure 1. HATA Model Graph for different Environments. 
from the environmental factors, the height of antenna plays significant role in determining the path loss.

\section{Path Loss Effect on BPSK Signal}

Information on a signal can be effectively transferred between the devices by modulating it according to the characteristic of the information. This work makes use of the Phase modulation for predicting the Path Loss in signal. In this the phase or the reference of the signal is changed to convey data ${ }^{6}$. The simplest of all the Phase Shift Keying is the Binary Phase Shift Keying. This Phase Shift keying uses two phases which are separated by $180^{\circ}$. Binary Phase Shift Keying is the robust of all Phase shift keying modulation scheme as it has a high tolerance level of noise on comparisons with other techniques. Binary Phase Shift Keying has wide application areas and is more commonly used for digital signal processing applications?. The required Binary Phase Shift Keying Signal is generated using the equation (4) as follows.

$$
S_{n}(t)=\sqrt{\left(\frac{2 E_{b}}{T_{b}}\right)} \cos \left(2 \pi f_{c} t+\pi(1-n)\right), n=0,1
$$

where, $S_{n}(t)$ is the Signal, $E_{b}$ is the Energy Per Bit, $T_{b}$ is the Bit Duration, $f_{c}$ is the frequency. Almost all the signals that we use today come with a certain amount of error because the signal is continuously exposed to the environmental noise and other disturbances. Since all the signals will generally consist of error in them, we have to consider certain amount of error along with the Binary Phase Shift Keying signal. The general form of Bit Error Rate for the Binary Phase Shift Keying Signal is given by the following equation (5).

$$
P_{b}=Q\left(\sqrt{\left(\frac{2 E_{b}}{N_{o}}\right)}\right)
$$

where, $\mathrm{P}_{\mathrm{b}}$ is the probability of bit error, $\mathrm{Q}$ is the probability that a single sample is taken and $\mathrm{N}_{\mathrm{o}}$ represents the spectral power density ${ }^{8}$. In addition to error a normal signal is also influenced by external noise. In this work we have taken a simple White Gaussian Noise as noise source and added to the signal. The following equation (6) denotes the general equation used to represent the White Gaussian Noise.

$$
p_{g}(\mathrm{z})=\left(\frac{1}{\sigma(\sqrt{2 \pi})}\right) e^{-\frac{(\mathrm{z}-\mu)^{2}}{2 \sigma^{2}}}
$$

where, $\mathrm{Z}$ represents Gray Level, $\mu$ represents the mean value, $\sigma$ represents the standard deviation. The generated signal is used to predict the effect of Path Loss using HATA's empirical formula and is shown graphically with the help of Okumura curves. Thus the BPSK signal generated by adding white Gaussian noise is used to identify path loss with the help of HATA empirical formulae for various terrains. The BPSK signal path loss at large urban, suburban, and rural areas are obtained using empirical formula for various distance and antenna height between different base stations. The graph shown in Figure 2 clearly shows

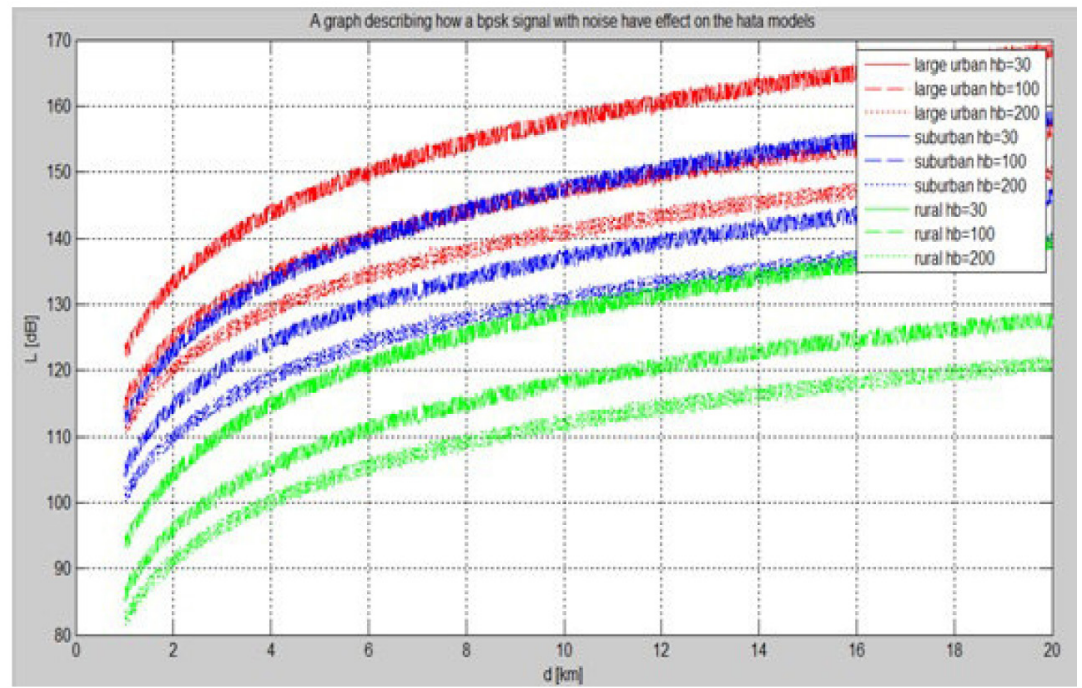

Figure 2. Path Loss Effect on a BPSK Signal to Noise. 
the difference between the normal signal and modulated signal. The modulated signal shows significant variations due to noise and is worst in case of large urban areas. The graph also indicates the disturbances in the signal due to the White Gaussian Noise ${ }^{8,9}$. The path loss in large urban region starts at $113 \mathrm{~dB}$ and for suburban and rural the value starts at $100 \mathrm{~dB}$ and $82 \mathrm{~dB}$ respectively. The difference is considerably more in case of large urban; therefore the effect of path loss due to environmental noise is significantly higher in large urban regions.

\section{Defining Path Loss Fuzzy Membership Function}

Fuzzy logic can be used to model anything, which cannot be defined in an absolute manner. It makes use of the fuzzy set ${ }^{10,11}$ which is not classical set by nature for defining the terms. It is just an extension of classical logic. In a more general way fuzzy logic is used for approximate reasoning. It can be used to represent multi valued logic. In a much more significant manner it is coextensive with the theory of fuzzy sets. The transaction of object from membership to nonmember ship is more gradual rather than abrupt. A Membership function usually characterizes the fuzzy set. A membership function usually defines the fuzzy set in the interval ${ }^{1}$. In this particular work, Triangular Membership is used for computing the membership value for the fuzzy set. These membership values are used for further computation in the fuzzy sets. Below is the formula for computing the Triangular Membership function.

$\mu_{F}(x, a, b, c)= \begin{cases}0, & \text { if } c<x<a \\ \frac{x-a}{b-a}, & \text { if } a \leq x \leq b \\ \frac{c-x}{c-b}, & \text { if } b \leq x \leq c\end{cases}$

The path loss values estimated using HATA model under various terrain regions by adjusting the parameters like antenna height and distance are given as input to the fuzzy system for determining the membership value of estimated path loss in large urban, urban and rural regions. Initially the large, urban, urban, and rural regions are modeled into urban, suburban and rural fuzzy sets with the help of $a, b$, and $c$ values determined from the set of data estimated using empirical formula. For this, the Minimum and maximum path loss values are identified from every set of data's estimated for different areas and consequently the mean value is computed. Thus, after determining a, b, and c, it is used to form fuzzy sets in different areas measured in urban, suburban and rural regions respectively. The triangle membership function is applied to determine the membership values for the urban, suburban and rural ${ }^{12,13}$. In all the cases a common distance (i.e.) $\mathrm{x}$ is assumed as input and maintained as constant for each of the calculation. Table below describes the value for $a, b, c$ for Urban, Sub Urban and Rural areas.

For any given value $\mathrm{x}$, the triangular function given in equation (7), is used to estimate the corresponding membership value $\mu_{F}$ in the defined fuzzy set. By the use of the above values the Triangular Membership Function can be calculated. Below are the graphs that are plotted by the use of calculated membership values.

The Figure 3 shows the fuzzy set membership plot for three different urban regions using the values given in table 1 . The path loss of urban region attains membership value $\mu_{F}=1$ at 146,131 , and 136 . The path loss range

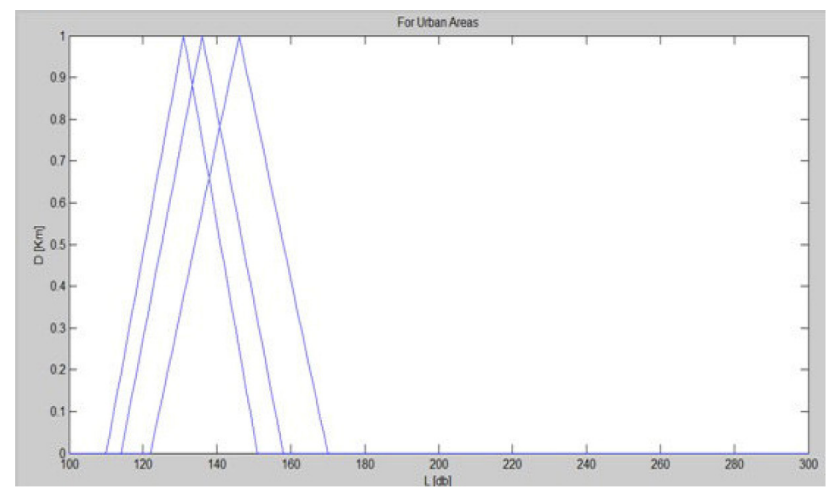

Figure 3. Urban areas.

Table 1. Membership Value for the different areas

\begin{tabular}{lcll}
\hline Membership Value/Areas A & b & \multicolumn{1}{l}{ c } \\
\hline Urban 1 & 122 & 146 & 170 \\
Urban 2 & 114 & 136 & 158 \\
Urban 3 & 110 & 131 & 151 \\
Sub Urban 1 & 112 & 136 & 159 \\
Sub Urban 2 & 104 & 126 & 148 \\
Sub Urban 3 & 100 & 121 & 141 \\
Rural 1 & 93 & 117 & 141 \\
Rural 2 & 85 & 107 & 129 \\
Rural 3 & 81 & 102 & 122 \\
\hline
\end{tabular}


associated with suburban and rural fuzzy sets are wider when compared with the urban fuzzy set.

Using the membership values we can compute the fuzzy sets, but apparently the fuzzy sets aren't much use in the real world, hence there is a need to convert the fuzzy set into the crisp set. Various Defuzzification methods are available to be converted the fuzzy values into crisp values; each of the defuzzification method has its use in the real world ${ }^{11}$. In this work Centre of Sums Defuzzification method is chosen for covert the fuzzy values to crisp values. Center of Sums is the fastest defuzzification method available today. It can be used for used for any kind of shape. Following is the formula used for computing the Centre of Sums.

$$
Z^{*}=\frac{\sum_{k=1}^{n} \mu c_{k}(z) \int_{z} \bar{Z} d z}{\sum_{k=1}^{n} \mu c_{k}(z) \int_{z} d z}
$$

Where, $\mathrm{Z}^{*}$ is the crisp output, $\mathrm{C}_{\mathrm{k}}$ is the area of the figure, $\mathrm{Z}$ is the center of the area, $\mu$ is the highest peak value.

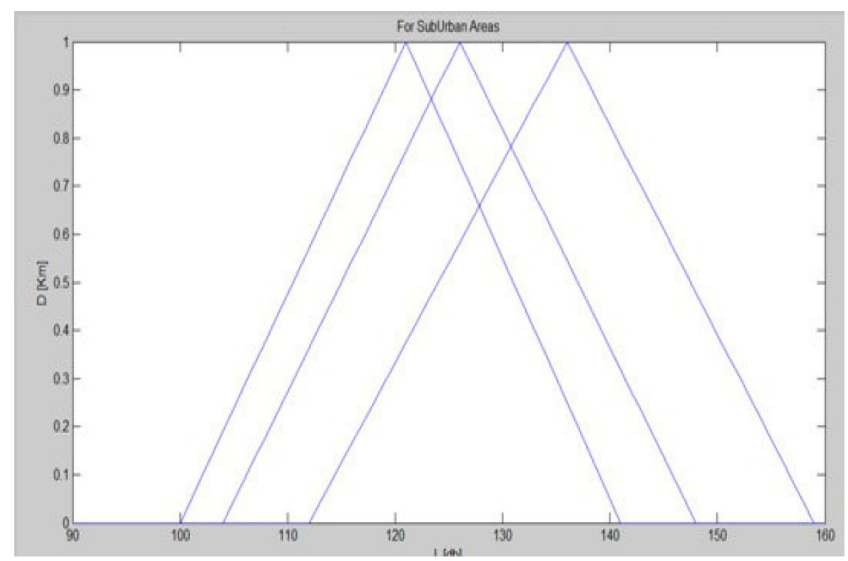

Figure 4. Sub Urban areas.

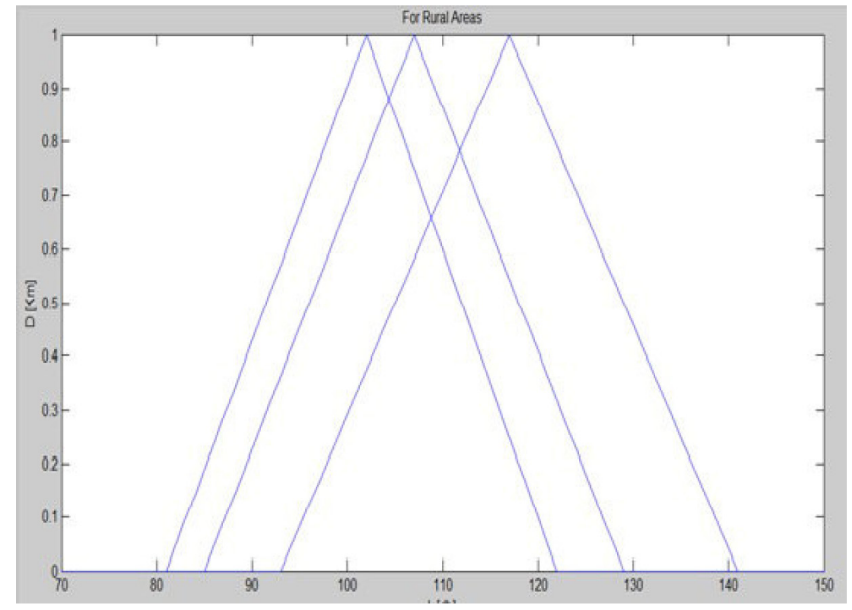

Figure 5. Rural area.

\section{Result and Discussion}

In this mode, it is seen that Path Loss occurs maximum at the Urban Areas and least in the rural areas (Figures 4 and 5). It increases constantly at the average rate of $10 \mathrm{db}$ per decade with the distance. Since urban areas are rather crowed compared to the Rural and Sub Urban areas, it's experience maximum effect of Path Loss. Using the HATA model the following Path Loss values were derived after Defuzzification.

The above data represents the Path Loss effect on Binary Phase Shift Keying signal. If the modulation technique changes the effect of Path Loss may vary accordingly.

\section{Conclusion}

The data obtained from the Path Loss model is represented in a Linear Regression using Fuzzy Logic. The data obtained from this model is based upon the distance between the Transmitter and the Receiver in different terrain. The HATA model provided with the basic outline of how to predict the Path Loss effectively and the use of Fuzzy Logic played a major role in converting the unreliable values to reliable ones. In a similar way this model can be used to effectively predict Path Loss in other kinds of modulated signal. In some cases this theoretical work has to comprised, but that is what defines engineering as a whole. Experimentation and Compromise define the basic character of an engineer. This work can be used effectively in a range of studies when the field values are not available.

\section{References}

1. Gupta A, Sharma SC, Vijay S, Gupta V. Secure path loss prediction using fuzzy logic approach. IEEE. 2008.

2. Rappaport TS. Wireless communication principles and practice, Pearson Education Pvt. Ltd; 2002.

3. Okumura Y. Field strength and its variability in UHF and VHF land mobile radio service. Review of Electrical Communication Laboratory; 1968.

Table 2. Path Loss values for different terrain type

\begin{tabular}{lc} 
Terrain Type & Fuzzy Path Loss Slope (n) \\
\hline Rural & 8 \\
Sub Urban & 28 \\
Urban & 38 \\
\hline
\end{tabular}


4. HATA M. Empirical formula for propagation loss in land mobile radio services. IEEE Trans Veh Tech; 1980.

5. Seidel SY, Rappaport TS. Site specific propagation prediction for wireless in building communication system design. IEEE Trans Veh Tech. 1994; 43:879-91.

6. Jorgen AB, Rappaport TS, Yoshida S. Propagation measurements and models for wireless communication channels. IEEE Communication Magazine; 1995.

7. Stuber GL. Principles of mobile communications. Kluwer Academic Publishers; 1996.

8. Lee WCY. Mobile Communication design fundamentals. 2nd Ed. John Wiely \& Sons, New York; 1993.
9. Seidel SY, Rappaport TS. $914 \mathrm{MHz}$ path loss prediction models for indoor wireless Communications in multi floored buildings. IEEE Trans Antenn Propag. 1992; 40:207-217.

10. Faruque S. Propagation prediction based on environmental classification and fuzzy logic approximation. IEEE Conference; 1996.

11. Ross TJ. Fuzzy logic with engineering application, John Wiley and Sons, Ltd; 2010.

12. Kosko B, Isaka S. Fuzzy logic. Sci Am; 1996: 76-81.

13. Kosko B. Fuzzy thinking. New York: Hyperion; 1993. 